\title{
Case Vectors: Spatial Representations of the Law using Document Embeddings
}

\author{
Elliott Ash and Daniel L. Chen*
}

June 29, 2018

\begin{abstract}
Recent work in natural language processing represents language objects (words and documents) as dense vectors that encode the relations between those objects. This paper explores the application of these methods to legal language, with the goal of understanding judicial reasoning and the relations between judges. In an application to federal appellate courts, we show that these vectors encode information that distinguishes courts, time, and legal topics. The vectors do not reveal spatial distinctions in terms of political party or law school attended, but they do highlight generational differences across judges. We conclude the paper by outlining a range of promising future applications of these methods.
\end{abstract}

\section{Introduction}

Law is embedded in language. In this chapter, we ask what can be gained by applying to the law new techniques from natural language processing that translate words and documents into vectors within a space. Vector representations of words and documents are informationdense-in the sense of retaining information about semantic content and meaning-while also being computationally tractable. This combination of information density and computational tractability opens up a wide potential realm of mathematical tools that can be used to generate quantitative and empirically testable insights into the law.

*Elliott Ash, Assistant Professor of Economics, University of Warwick, e.ash@warwick.ac.uk. Daniel L. Chen, Professor of Economics, University of Toulouse, daniel.chen@iast.fr. We thank Brenton Arnaboldi, David Cai, Matthew Willian, and Lihan Yao for helpful research assistance. 
This new approach to legal studies addresses the shortcomings of existing methods for studying legal language. Because law consists of text, research methods based on formal math and numerical data are somewhat limited in the questions that can be asked. The formal theory literature has come at the law metaphorically. This case-space literature, in particular, treats the law spatially, where the law separates the fact space into "liable" and "not liable" or "guilty" and "not guilty." The case space models give us some intuition into the legal reasoning process. But they have been somewhat limited empirically because it has been infeasible to measure the legal case space. The traditional empirical legal studies literature has relied on small-scale data sets, where legal variables are manually coded (e.g. Songer and Haire, 1992).

Meanwhile, recent work in computational linguistics has made breakthroughs in vector representations of language (Blei, 2012; Mikolov et al., 2013; Jurafsky and Martin, 2014). For example, the success of Google's Word2Vec algorithm is that it "learns" the conceptual relations between words; a trained model can produce synonyms, antonyms, and analogies for any given word (Mikolov et al., 2013; Levy et al., 2015). These "word embeddings," as the word vectors have come to be called, serve well as features in down-stream prediction tasks by encoding a good deal of information in relatively rare word features. More recently, "document embeddings" have built upon the success of word embeddings to represent words and documents in a joint geometric space (Le and Mikolov, 2014). Like word embeddings, these document embeddings have advantages in terms of interpretability and serve well in prediction and classification tasks.

An active literature in computational legal studies has begun to apply these methods to legal documents. Livermore et al. (2016) use a topic model to understand agenda formation on the U.S. Supreme Court. Leibon et al. (2018) use a network model to represent the geometric relations between U.S. Supreme Court cases. Ganglmair and Wardlaw (2017) apply a topic model to debt contracts, while Ash et al. (2018b) apply one to labor union contracts.

This paper expands on this work in the context of the universe of U.S. Supreme Court and U.S. Circuit Court cases for the years 1887 through 2013. We construct document embeddings for each opinion in the corpus. We then construct judge vectors by taking the average of the document embeddings for the cases authored by the judge. These case vectors are used to analyze the geometry of federal appellate caselaw.

We ask whether the information recovered by our model provides a meaningful signal

\footnotetext{
${ }^{1}$ Cameron and Kornhauser (2017) provide a recent review of this literature.
} 
about the legal content in cases. We find that spatial clustering in these embeddings encode differences between cases on different courts, between cases in different years, and between cases in different legal topics. The vectors can also discriminate judges baes on birth cohorts, but does not do well in encoding the partisan affiliation of judges or law school attended. We also demonstrate that the vectors can show which judges are similar to each other in their legal writing.

In the concluding section we outline a range of potential future applications for the use of embeddings models in computational analysis of law. First, structured embeddings could be used to explicitly model the relations between judges, between courts, or over time. Second, citation embeddings might be used to identify similar cases based on how often they are cited together. Third, embeddings might shed light on differences across judges in sentiment toward policies or social groups. Fourth, we could construct judge embeddings based on their their predictiveness for case outcomes, rather than just the language features.

\section{Embeddings Models and the Law}

A first-order problem in empirical analysis of text data is the high dimensionality of text. There are an arbitrary number of approaches for representing plain text as data. One must trade off informativeness, interpretability, and computational tractability (Ash, 2017). For example, one could represent a document as a frequency distribution over words. But with a large vocabulary, say 20,000 words, a document is still a high-dimensional vector.

Word embeddings came about as a dimension reduction approach in deep learning models for prediction tasks in computational linguistics (Mikolov et al., 2013). Such a prediction task would include, for example, predicting the next word in a sequence given a set of words in a sentence. To that end, the model represents a word as a small and dense vector (say 100 dimensions). Initially, words are randomly distributed across the vector space. But the word locations then become features in a learning model; the word locations then move around during training to improve performance on a prediction task. In natural language settings, this process typically leads to words clustering near similar words.

Document embeddings, such as Le and Mikolov's (2014) paragraph vectors, use a separate embedding layer for both the word and the document to solve the prediction task. These models locate documents in a vector space, where documents that contain similar language tend to be located near to each other in the space. Embedding models are different from topic models (e.g. Blei, 2012) because the dimensions have a spatial interpretation, rather 
than a topic-share interpretation. Document embeddings have become popular because the spatial relations between the trained embeddings encode useful and meaningful information (Levy et al., 2015).

To illustrate, a word embedding can identify similar words in the vocabulary. For example, "judge" might be close to "jury" but far away from "flowerpot." Similarly, a document embedding can identify similar cases in a corpus of decisions based on use of similar language. For example, Engel v. Vitale (1962) might be spatially close to Everson v. Board of Education (1947), since they are both early U.S. Supreme Court decisions that deal with religious freedoms in the states. Finally, a judge embedding constructed from these documents could be used to identify similar judges in the legal system. For example, the closest judge to Antonin Scalia might be Clarence Thomas.

A more intriguing exercise is to think about analogies. A well-known example is that word embeddings "know" that "man" is to "woman" as "king" is to "queen," through the vector algebra king - man + woman = queen (Mikolov et al., 2013; Ash, 2016). Similarly, a document embedding could say something like "Everson vs. Board of Education is to Engel v. Vitale as Griswold v. Connecticut is to Roe v. Wade." These cases share an analogical relation, in that the latter case is a related application of the constitutional principle articulated in the former case. In the vector math, that would be represented as Everson - Engel + Griswold $=$ Roe. Finally, a judge embedding could say something like "Scalia is to Thomas as Ginsburg is to Breyer," in the sense that Scalia - Thomas + Breyer $=$ Ginsburg.

In the case of word embeddings, the directions in the embedding space often encode semantic meaning. For example, Bolukbasi et al. (2016) show that there is a vector direction for gender in the embedding space. One can also typically isolate directions for time, singular vs plural, etc. In the legal case, we would be interested in isolating directions for legal and political concepts and distinctions. For example, might there be a direction for liberal vs conservative, or procedural vs substantive? Are there directions or clusters for originalists, or pragmatists, or economic analysis?

The use of embedding layers for informative dimension reduction has much untapped potential in empirical social science (see, e.g., Rudolph et al., 2017).

\section{Application to Federal Appellate Courts}

This section illustrates the use of document embeddings in the federal appellate courts. 


\subsection{Data and Documents}

The analysis utilizes a corpus of all U.S. Supreme Court cases, and all U.S. Circuit Court cases, for the years 1887 through 2013. We have detailed metadata for each opinion; we mainly use the court, date, case topic, and authoring judge. For case topic, we use the 7category "General Issue" designation coded for Donald Songer's Court of Appeals Database. The cases are linked to biographical information on the judges obtained from the Federal Judicial Center. This includes birth date, gender, and political affiliation of appointing president.

We also have the full text of the cases. We remove HTML markup and citations. We then have each case as a list of tokens. These tokens provide the inputs for the embeddings model.

\subsection{Construction of Document Vectors}

The next step is to construct document vectors for each case $i$. The model we use is Doc2Vec (Le and Mikolov, 2014), implemented in the Python package gensim. The objective function solved by this model is to iterate over the corpus and try to predict a given word using its context (a window of neighboring words), as well as a bag-of-words representation of the whole document. The model uses an embedding layer for the context features and the document features. Therefore the geometric location of documents encodes predictive information for the context-specific frequencies of words in the document.

We feed the case documents in random order into Doc2Vec, using standard parameter choices. We used the distributed bag-of-words model over the distributed memory model, with 200 dimensions per document vector. Other parameter choices include a context window of size 10, capping the vocabulary at 100,000 words (based on document frequency), and excluding documents shorter than $\mathbf{4 0}$ words in length. The model iterates through the corpus in random order for five epochs.

\subsection{Vector Centering and Aggregation}

We now have a set of vectors $\vec{i}$ for each case $i$. Following the advice of the embeddings literature, ${ }^{2}$ we normalized each vector to length one. Each case has an authoring judge $j$, working in court $c$ at year $t$. Besides author and time, the other metadata feature is the case topic $k$.

\footnotetext{
2See Omer Levy, "Should I do normalization to word embeddings?". Quora, 7 November 2015.
} 
For visualization and other analysis we would like to center and aggregate the document vectors in several ways. Let $I_{j}$ be the set of cases authored by $j$. Let $I_{j t}$ be the set of cases authored by $j$ at year $t$. One could construct a vector representation for a judge using

$$
\vec{j}=\frac{1}{\left|I_{j}\right|} \sum_{i \in I_{j}} \vec{i}
$$

where $|\cdot|$ gives the count of the set. Similarly, the vector for judge $j$ at year $t$ would be given by

$$
\overrightarrow{j t}=\frac{1}{\left|I_{j t}\right|} \sum_{i \in I_{j t}} \vec{i}
$$

and the vector for all cases on topic $k$ in court $c$ during year $t$ would be given by

$$
c \vec{k} t=\frac{1}{\left|I_{c k t}\right|} \sum_{i \in I_{c k t}} \vec{i}
$$

Meanwhile, the same notation and corresponding aggregation formula could be used to construct a vector for a year, $\vec{t}$, for a court $\vec{c}$, for a topic $\vec{k}$, or for the cases in court $c$ during a particular year $t, \overrightarrow{c t}$.

We are interested in recovering the ideological component of the judge vectors. Therefore we explore the following steps to center the document vectors before aggregating. Represent the year-centered vector for case $i$ as $\vec{i}_{t}=\vec{i}-\vec{t}_{i}$, where $\vec{t}_{i}$ corresponds to the average vector for all cases in the same year as $i$. Similarly, let a subscripted judge vector $\overrightarrow{j_{t}}$ be defined as

$$
\overrightarrow{j_{t}}=\frac{1}{\left|I_{j}\right|} \sum_{i \in I_{j}} \vec{i}_{t}
$$

the average for judge $j$ of the year-centered vectors $\vec{i}_{t}$.

The preferred centering specification depends on the context of the analysis. We center by interacted groups, in particular. In the results below, we variously center by topic-year $\overrightarrow{k t}$, by court-year $\overrightarrow{k t}$, and by court-topic-year $c \overrightarrow{k t}$. Only after this centering step do we aggregate by judge and perform analysis of the spatial relations between vectors. The hope is that the remaining spatial variation is purged of court-specific, topic-specific, and yearspecific differences in language. The remaining variation will provide a cleaner summary of the ideological differences between judges.

Here we have used the unweighted average of the case vectors, where each case is weighted equally. Future work might explore the use of other weighting schemes. A sensible 
alternative would be to weight the cases by their length (in words or sentences), for example. In addition, it would be reasonable to weight the cases by the number of citations they later received - as a proxy for importance.

\subsection{Visual Structure of Case Vectors and Judge Vectors}

In this section we present a variety of visualizations to understand better the spatial relationships encoded by our case vectors and judge vectors. Our visualization methods is a t-SNE plot (Maaten and Hinton, 2008), which projects the vectors down to two dimensions for visualization purposes. We use t-SNE plots, rather than principal components, because the dimension reduction algorithm is designed to project data while preserving relative distance between points. The dots represent vectors, and the colors/labels represent groupings.

We begin by exploring the institutional, temporal, and judge-level features encoded in the vectors. For Figure 1, we centered the case vectors by topic interacted with year, as described in Section 3.3. We then averaged by judge and plotted the judge vectors. The vectors are labeled by court. One can see that, conditional on topic and year, the document vectors separate the courts quite well. This is consistent with systematic differences in legal language across courts, conditional on topic and year, being captured by the embedding.

For Figure 2, we centered on court interacted with topic. We then average by court-year and plotted the court-year-level averaged vectors. We labeled and colored by the decade the case was published. One can see a steady linear development of case law across the geometric space. This shows that, controlling for court and topic factors, the embedding captures systematic differences in language across time.

For Figure 3, we centered on judge interacted with year; this residualizes out any judgelevel time-varying components of language. We then averaged and plotted by topic-year. The labels and colors distinguish the seven-digit general issue topic. We can see that the document embeddings discriminate topics, effectively capturing differences in language across recognized issue areas.

Next we look at whether the vectorized language in the case vectors encodes information about judge characteristics. For Figure 4, we centered on an interacted groupings for court, topic, and year. This centering controls for any time-varying topic and court level language variation. We then averaged by judge and plotted the judge vectors. The labels and colors are by political party - Democrat or Republican. These are randomly distributed across the graph. It appears that the language features encoded by the document embeddings are not informative about political party. One interpretation is that judicial language is not very 
Figure 1: Centered by Topic-Year, Averaged by Judge, Labeled by Court

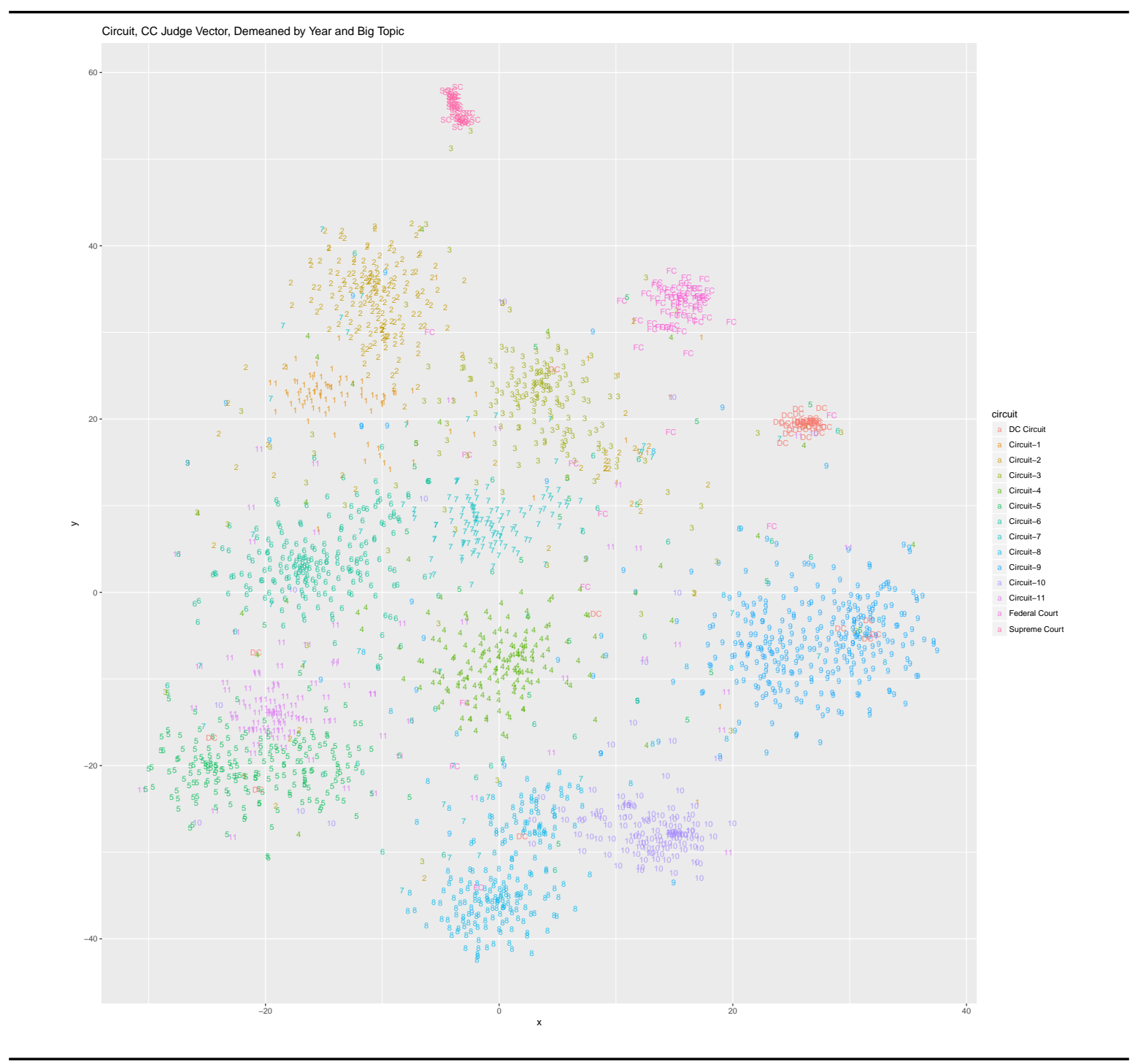


Figure 2: Centered by Court-Topic, Averaged by Court-Year, Labeled by Decade

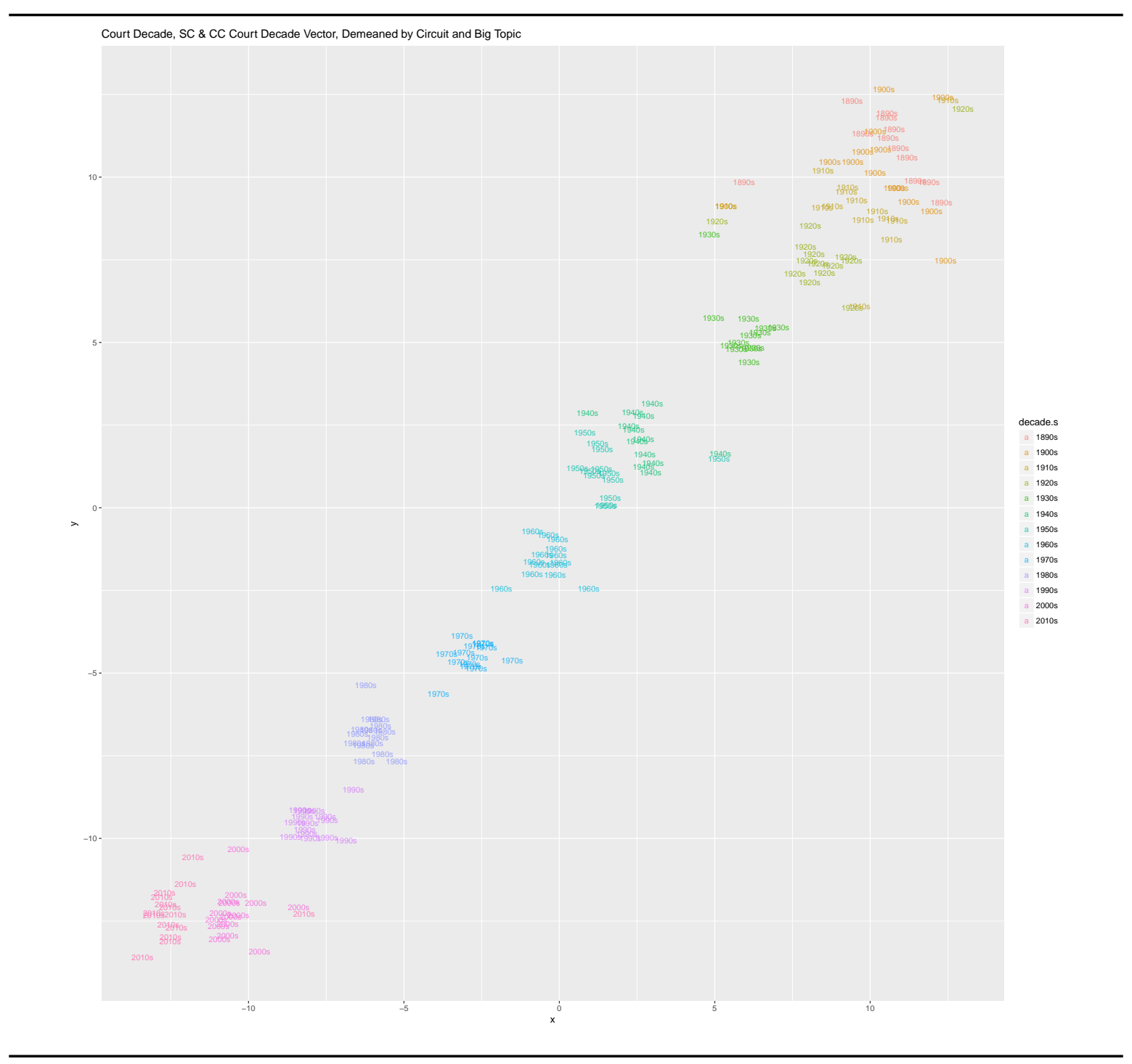


Figure 3: Centered by Judge-Year, Averaged by Topic-Year, Labeled by Topic

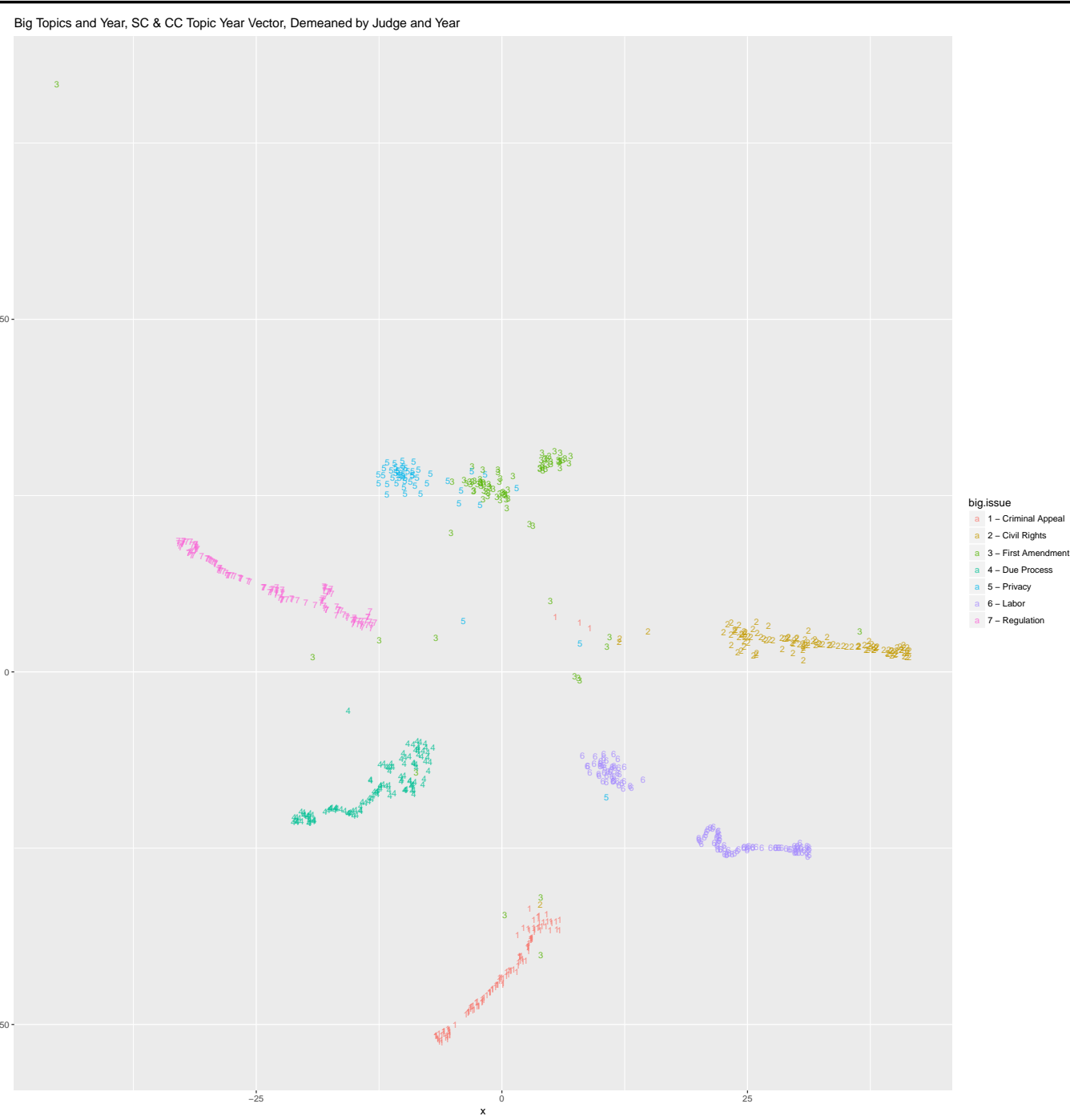


politicized (related to the result in Ash et al. (2017) that judicial language is less polarized than congressional language). Another possibility is that our representation of language is not rich enough to encode ideological content. Richer representations, such as those constructed from grammatical relations between words (Levy and Goldberg, 2014), may be needed.

Figure 5 considers another judicial biographical feature: birth cohort. As before, we centered on court-topic-year and averaged/plotted by judge. In this case, the labels and colors are by birth cohort decade (1910s through 1950s). In stark contrast to political party, there is clear segmentation across the geometric space across cohorts. Remember that this is conditioned on court-topic-year, so is not driven by time trends over the sample. The vectorized language recovers differences in the legal language used by judges from different generations.

Finally, for Figure 6, we consider law school attended as a final source of linguistic differences across judges. Conditional on court, topic, and year, we see apparent random distributions across the space in terms of law school. As with political party, it seems like language or ideological differences by school do not show up in the vectors. Again, this may be due to ideologically distinctive embeddings requiring a richer representation of language than that used here.

\subsection{Analysis of Relations Between Judges}

This section uses our vector representation of judges to produce a similarity metric between courts and judges. We adopt a measure of vector similarity that is used often for document classification. The cosine similarity between two vectors,

$$
s(\vec{v}, \vec{w})=\frac{\vec{v} \cdot \vec{w}}{\|\vec{v}\|\|\vec{w}\|},
$$

which is equal to one minus the cosine of the angle between the vectors. It takes a value between -1 and 1 . In the case of word embeddings, high similarity means that the words are often used in similar language contexts.

In the case of judges, we can say that similarities approaching one mean that the judges tend to use similar language in their opinions. Similarities approaching -1 meaning the judges rarely use the same language. Similarities near zero mean that the judges are as similar to each other as would be expected from two randomly selected judges in the population.

First we look at similarity between court vectors to complement the spatial representation 
Figure 4: Centered by Court-Topic-Year, Averaged by Judge, Labeled by Political Party

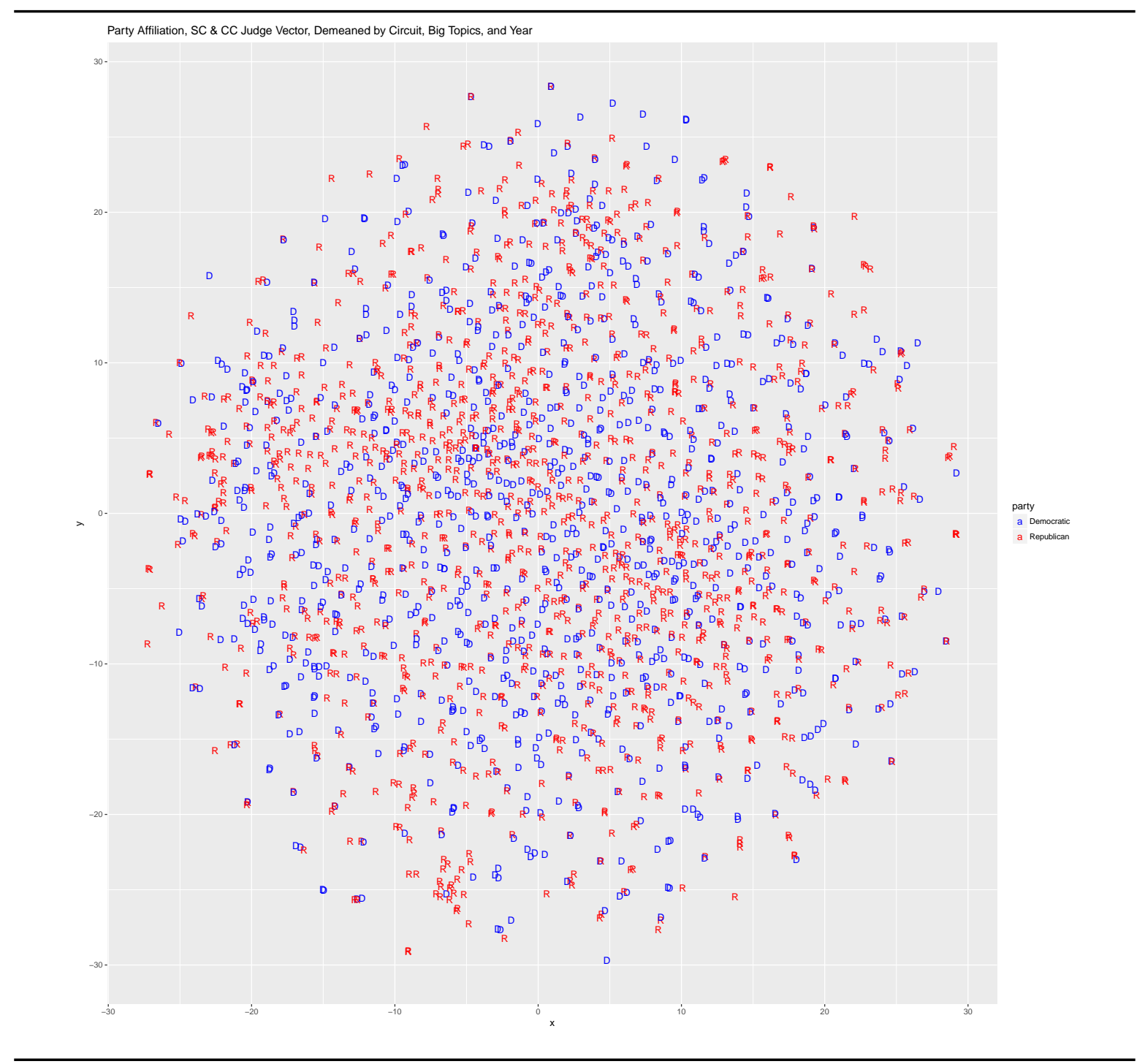


Figure 5: Centered by Court-Topic-Year, Averaged by Judge, Labeled by Judge Birth Cohort

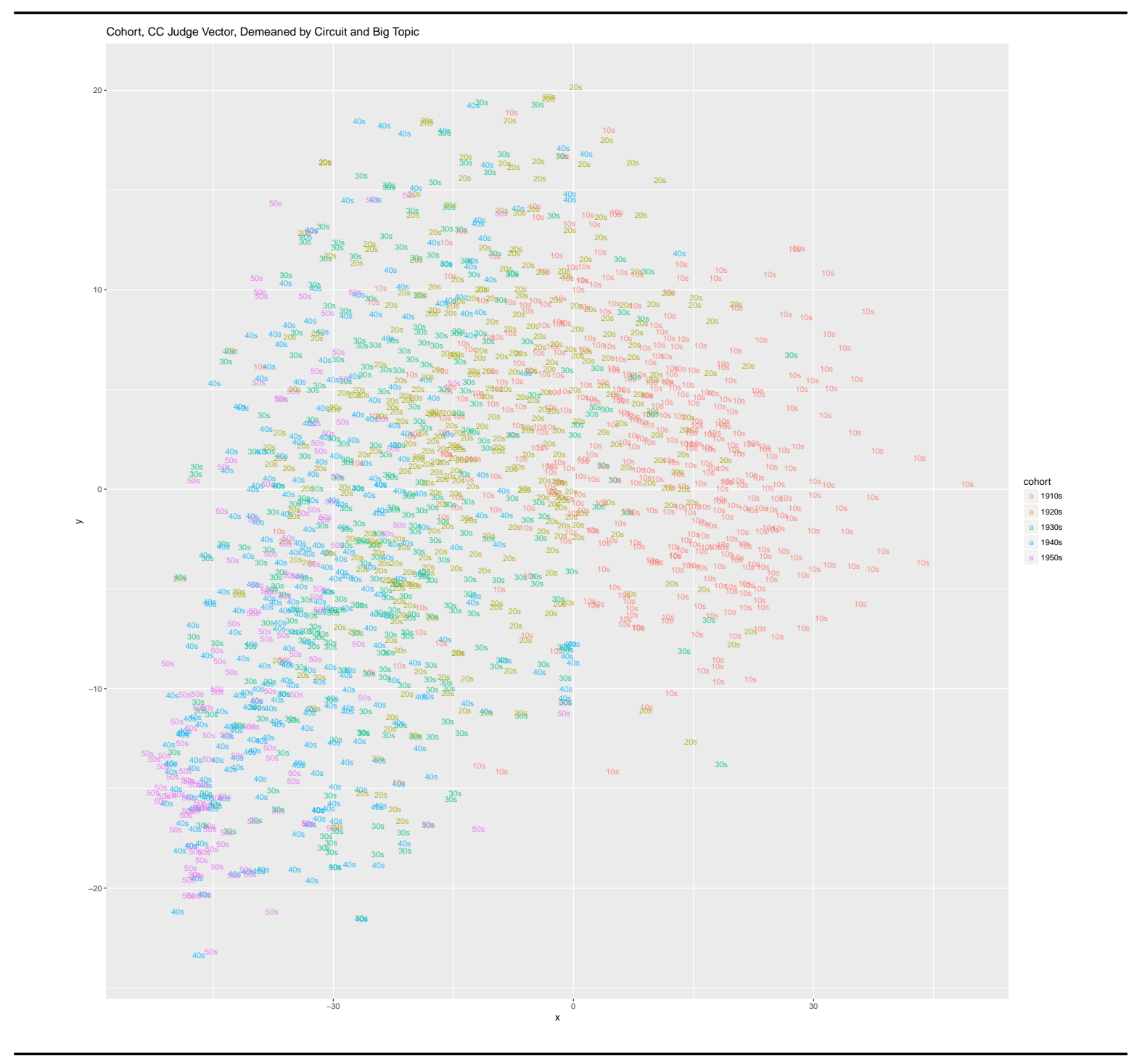


Figure 6: Centered by Court-Topic-Year, Averaged by Judge, Labeled by Law School Attended

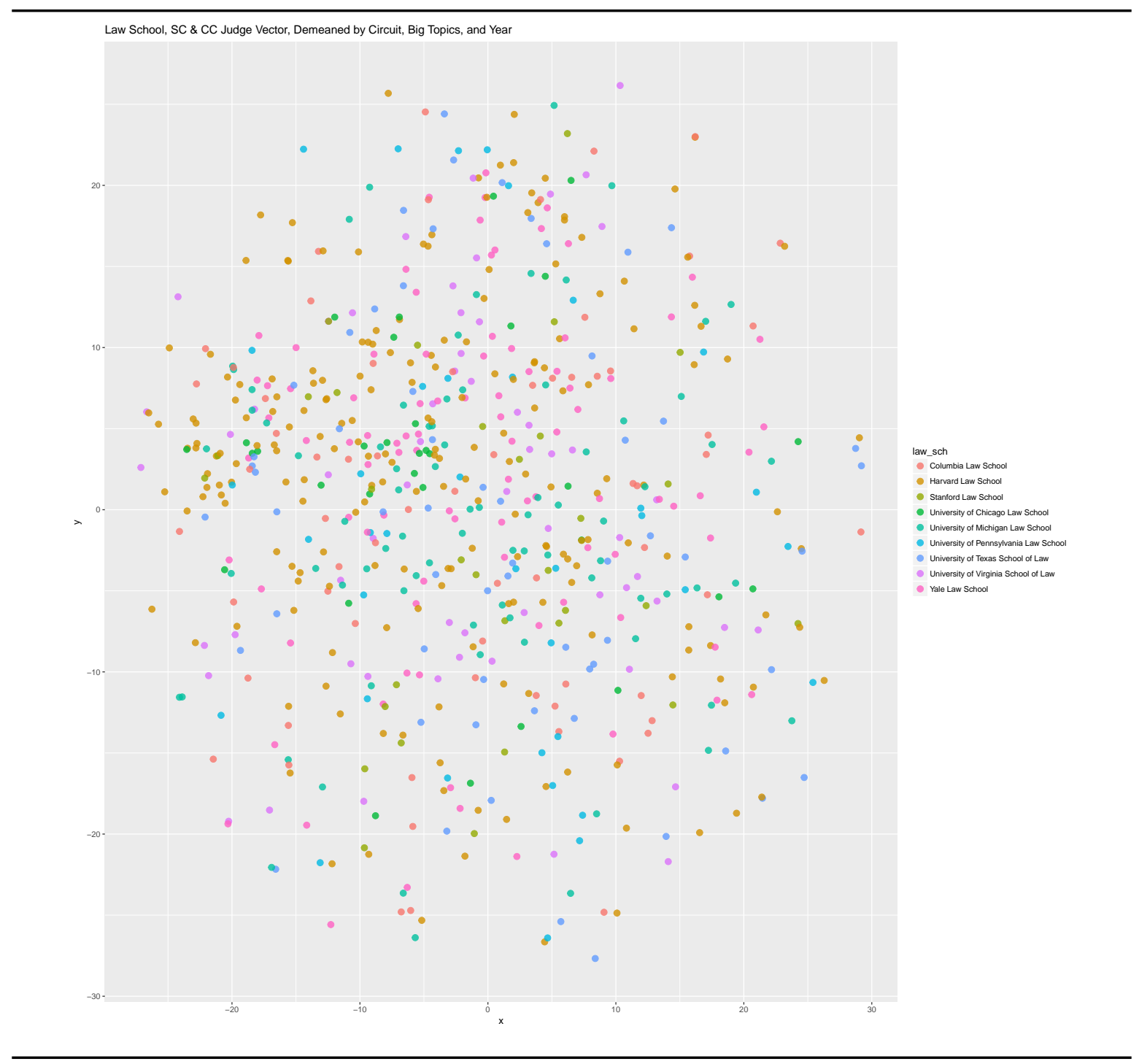


Table 1: Pair-Wise Similarities Between Federal Appellate Courts

\begin{tabular}{|c|c|c|c|c|c|c|c|c|c|c|c|c|c|c|}
\hline & scotus & $1^{\text {st }}$ Circ. & $2^{\text {nd }} \cdot$ Circ. & $3^{\text {rd }}$. Circ. & $4^{\text {th }}$ Circ. & $5^{\text {th }}$ Circ. & $6^{\text {th }}$ Circ. & $7^{\text {th }}$ Circ. & $8^{\text {th }}$ Circ. & $9^{\text {th }}$ Circ. & $10^{\text {th }}$ Circ. & 11.th Circ. & D.C. Circ. & Fed. Circ. \\
\hline scotus & 1.000 & & & & & & & & & & & & & \\
\hline $1^{\text {st }}$ Circ. & 0.022 & 1.000 & & & & & & & & & & & & \\
\hline $2^{\text {nd }}$. Circ. & -0.008 & 0.302 & 1.000 & & & & & & & & & & & \\
\hline $3^{\text {rd }}$. Circ. & -0.001 & 0.135 & 0.207 & 1.000 & & & & & & & & & & \\
\hline $4^{\text {th }}$ Circ. & -0.045 & -0.045 & -0.081 & 0.126 & 1.000 & & & & & & & & & \\
\hline $5^{\text {th }}$ Circ. & -0.105 & -0.196 & -0.298 & -0.269 & 0.038 & 1.000 & & & & & & & & \\
\hline $6^{\text {th }}$ Circ. & -0.074 & -0.185 & -0.148 & 0.009 & 0.069 & -0.107 & 1.000 & & & & & & & \\
\hline $7^{\text {th }}$ Circ. & -0.097 & -0.052 & -0.014 & -0.055 & -0.162 & -0.257 & 0.029 & 1.000 & & & & & & \\
\hline $8^{\text {th }}$ Circ. & -0.137 & -0.215 & -0.296 & -0.214 & -0.150 & -0.184 & 0.050 & -0.022 & 1.000 & & & & & \\
\hline $9^{\text {th }}$ Circ. & 0.039 & -0.137 & -0.140 & -0.182 & -0.147 & -0.121 & -0.220 & -0.265 & -0.150 & 1.000 & & & & \\
\hline $10^{\text {th }}$ Circ. & -0.111 & -0.249 & -0.361 & -0.179 & -0.189 & 0.017 & 0.006 & -0.158 & 0.218 & 0.042 & 1.000 & & & \\
\hline 11.th Circ. & -0.086 & -0.191 & -0.240 & -0.215 & 0.067 & 0.713 & -0.039 & -0.224 & -0.192 & -0.084 & 0.026 & 1.000 & & \\
\hline D.C. Circ. & 0.846 & -0.085 & -0.058 & 0.011 & -0.010 & -0.062 & -0.097 & -0.177 & -0.111 & 0.067 & -0.025 & 0.011 & 1.000 & \\
\hline Fed. Circ. & 0.178 & 0.200 & 0.132 & 0.116 & 0.124 & -0.150 & -0.154 & -0.082 & -0.255 & -0.116 & -0.260 & -0.181 & 0.094 & 1.000 \\
\hline
\end{tabular}

in Figure 1. We centered the vectors by topic and year, and then aggregated by court. We then computed the pair-wise similarities between the court vectors. These are reported in Table 1.

The colors provide a gradient for similarity, with green meaning the courts are relatively similar and red meaning they are relatively dissimilar. The table has some interesting features. First, the D.C. Circuit is most similar to the Supreme Court of the United States, which is intuitive since they are both located in Washington, D.C. and focus on issues of federal government functioning such as separation of powers. Second, the 11th circuit is similar to the 5th circuit, which is intuitive since the 11th Circuit used to be a part of the 5th Circuit and they share many legal precedents.

Next we look at similarity between judge vectors. Starting with the Supreme Court, we center the document vectors on topic, and year. Then we take the average of these centered vectors by judge as our representation of judge writing, reasoning, and beliefs. Table 2 (continued in Table 3) reports the pair-wise similarities between a selection of recently sitting Supreme Court judges. Overall, there are limited immediate insights and the results are mixed. For example, it is intuitive that Scalia is close to Thomas. But counter-intuitively, Scalia is even closer to Souter, Stevens, and O'Connor. Another example: Intuitively, Brennan is close to Thurgood Marshall; but counter-intuitively, he is closer to White and Stewart. Overall, the judge vectors do not seem to encode similarities between Supreme court judges very well. This may be due to the relatively few decisions that they author. In particular, the relative dissimilarity between Kagan and most other justices is 
likely due to her having only a handful of decisions in the corpus.

One interesting feature of our model is that it represents both circuit court judges and supreme court judges in the same geometric space. As done previously, we center all the document vectors on court, topic, and year. We then aggregate by judge. For Table 4, we computed the vector similarity between each circuit court judge and each supreme court judge. We then ranked the circuit court judges by this similarity. The table shows, for each supreme court judge, the top 5 circuit court judges on this ranking. As with the pairwise similarities between supreme court judges, these rankings are not particularly intuitive or informative. Understanding the limitations of these types of models is important for future research. An important factor is that we use a bag-of-words model, and ideological differences between judgs may be mostly encoded in phrases.

A possible reason for the lackluster results in the Supreme Court is that the justice vectors may not be well defined due to the small number of opinions they publish. Therefore we round out this analysis by looking at a notable circuit court judge, Richard A. Posner. The document vectors are de-meaned by court, year, and topic. Then they are aggregated by judge. Then we rank all circuit court judges by the similarity of their vector to Posner's vector. These are reported in Table 5. Interestingly, the most similar judge is Frank Easterbrook, who, like Posner is known for the use of economic analysis in opinions. Stephen Breyer has a published article in The Economic Journal on "economic reasoning and judicial review" (Breyer, 2009). Posner has a conservative reputation, and we see other conservative judges such as Neil Gorsuch and Antonin Scalia. Henry Friendly makes an appearance - he is a well-known pragmatist, as is Posner. Finally, Michael McConnell co-write law articles with Posner. The document vectors, as trained in this example, are much more informative about the connections between circuit court judges than between Supreme Court judges.

\section{Discussion of Parallel and Future Work}

We conclude with a discussion of how future work could adapt these embeddings models for empirical analysis of law.

\subsection{Structured Group Embeddings}

The document embeddings developed in the previous section were static, and did not ex-

plicitly model a time component. In addition, they only encoded judge identity by taking the average of a judge's document vectors. Recent work in embeddings models seeks to 


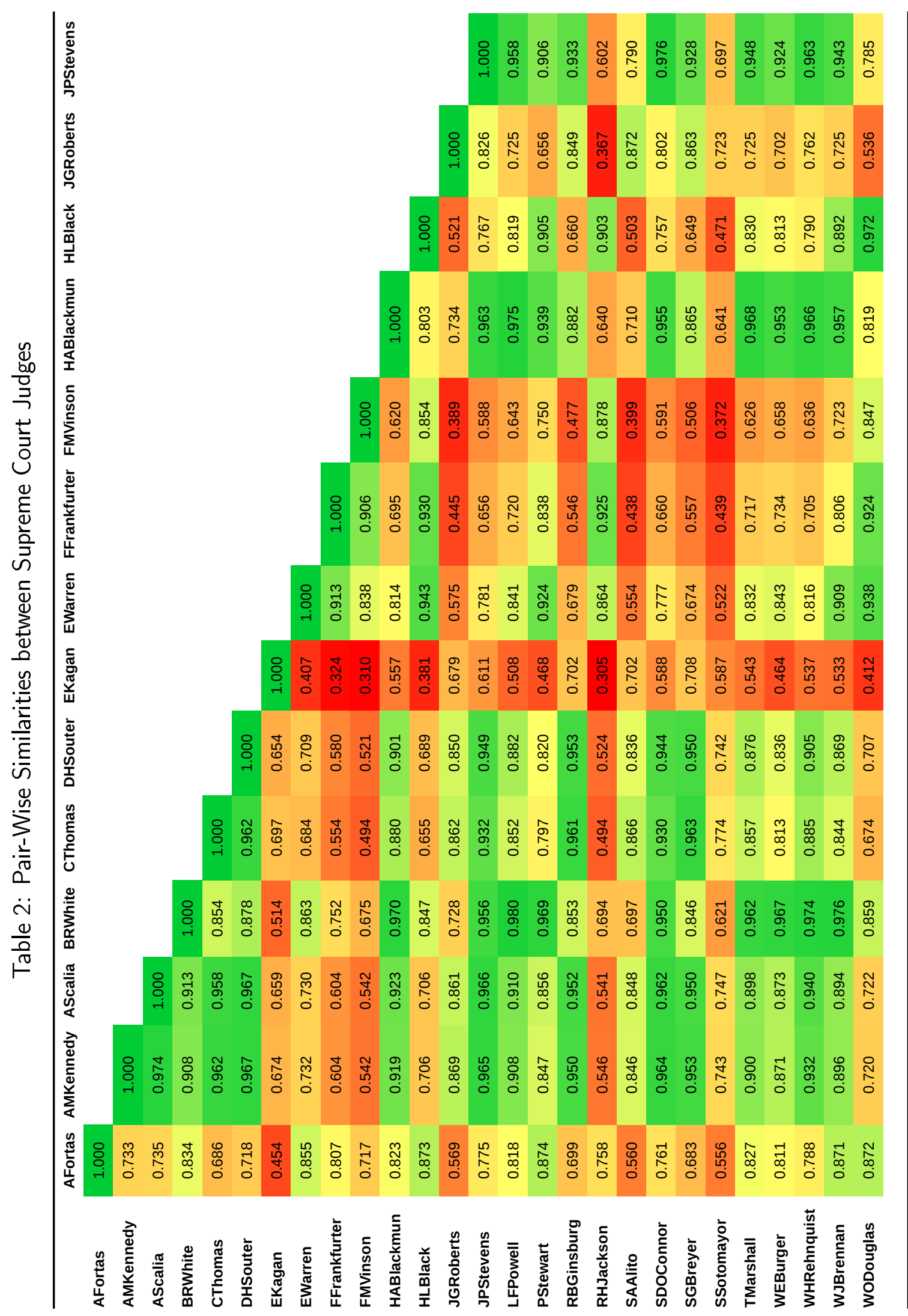


Table 3: Pair-Wise Similarities between Supreme Court Judges (cont.)

\begin{tabular}{|c|c|c|c|c|c|c|c|c|c|c|c|c|c|}
\hline & LFPowell & PStewart & RBGinsburg & RHJackson & SAAlito & SDOConnor & SGBreyer & SSotomayor & TMarshall & WEBurger & WHRehnquist & WJBrennan & wODouglas \\
\hline LFPowell & 1.000 & & & & & & & & & & & & \\
\hline PStewart & 0.954 & 1.000 & & & & & & & & & & & \\
\hline RBGinsburg & 0.854 & 0.794 & 1.000 & & & & & & & & & & \\
\hline RHJackson & 0.669 & 0.772 & 0.493 & 1.000 & & & & & & & & & \\
\hline SAAlito & 0.688 & 0.642 & 0.860 & 0.369 & 1.000 & & & & & & & & \\
\hline SDOConnor & 0.946 & 0.898 & 0.921 & 0.597 & 0.778 & 1.000 & & & & & & & \\
\hline SGBreyer & 0.841 & 0.786 & 0.959 & 0.492 & 0.885 & 0.926 & 1.000 & & & & & & \\
\hline SSotomayor & 0.621 & 0.598 & 0.745 & 0.365 & 0.741 & 0.708 & 0.744 & 1.000 & & & & & \\
\hline TMarshall & 0.961 & 0.936 & 0.868 & 0.666 & 0.690 & 0.926 & 0.845 & 0.605 & 1.000 & & & & \\
\hline WEBurger & 0.971 & 0.950 & 0.809 & 0.672 & 0.665 & 0.921 & 0.801 & 0.613 & 0.931 & 1.000 & & & \\
\hline WHRehnquist & 0.970 & 0.939 & 0.876 & 0.637 & 0.729 & 0.972 & 0.873 & 0.672 & 0.937 & 0.964 & 1.000 & & \\
\hline WJBrennan & 0.968 & 0.971 & 0.849 & 0.749 & 0.691 & 0.929 & 0.841 & 0.620 & 0.964 & 0.950 & 0.946 & 1.000 & \\
\hline wODouglas & 0.833 & 0.913 & 0.681 & 0.905 & 0.525 & 0.766 & 0.673 & 0.488 & 0.851 & 0.820 & 0.796 & 0.904 & 1.000 \\
\hline
\end{tabular}

include these relations more flexibly and elegantly as a part of the data generating process. Rudolph and Blei (2017) provide a model for learning dynamic embeddings, and look at how language has changed over time in the U.S. Congress over the last century. Rudolph et al. (2017) provide a model for structured group embeddings, and allow word and document vectors to have a group component and an individual component.

In parallel work, we found difficulties in initial applications of structured embeddings to judge groups (Ash et al., 2018a). Word similarities seem to be highly sensitive to model parameters. Systematic differences in word similarities between Republican and Democrat judges can flip based on the embedding dimension and vocabulary size, for example.

\subsection{Vectorization of Citation Networks}

The approach above used only the language of opinions to represent legal ideas. But we all know that in a common law system, the previous cases cited are a major expression of the ideological content of a decision. In parallel work we are experimenting with enriching case representations with information from the citation graph (e.g. Ash et al., 2017). Citations could be included as features in the document embedding, which might reveal more differences, such as those between political parties. This work might explore recent advances in vectorizing networks, such as node2vec (Grover and Leskovec, 2016).

Another application of embedding models to citations is based on Rudolph et al. (2017), where the model predicts occurrence of a product in a grocery shopping cart based on the co-occurrence of other products. In a forthcoming working paper, we treat cases as a 
Table 4: Most Similar Circuit Court Judges to each Supreme Court Judge

\begin{tabular}{|c|c|c|}
\hline W E Burger & A M Kennedy & A Scalia \\
\hline MARBLEY, ALGENON L. & SARGUS, EDMUND A., JR. & ROBERTS, VICTORIA A. \\
\hline MURRAY, HERBERT F. & NICKERSON, EUGENE H. & VANCE, SARAH SAVOIA \\
\hline HULL, THOMAS GRAY & NOTTINGHAM, EDWARD WILLIS, JR. & LAKE, SIMEON TIMOTHY, III \\
\hline O'SULLIVAN, CLIFFORD & PECK, JOHN WELD & SHAW, CHARLES A. \\
\hline DOTY, DAVID S. & JOHNSEN, HARVEY & O'NEILL, THOMAS N., JR. \\
\hline C Thomas & D H Souter & E Warren \\
\hline KEELEY, IRENE PATRICIA M. & MOTZ, DIANA GRIBBON & ZAVATT, JOSEPH C. \\
\hline FISHER, JOE J. & MARRERO, VICTOR & DYER, DAVID PATTERSON \\
\hline MCCORD, LEON & DIAMOND, GUSTAVE & SWAN, THOMAS W. \\
\hline SMITH, WILLIAM F. & WANGELIN, H. KENNETH & WHITAKER, SAMUEL \\
\hline KEENAN, BARBARA MILANO & BOOCHEVER, ROBERT & MCCORD, LEON \\
\hline H A Blackmun & H L Black & J G Roberts \\
\hline CORDOVA, VALDEMAR A. & THOMPSON, JOSEPH W. & STEIN, SIDNEY H. \\
\hline SINGLETON, JOHN V., JR. & MINER, ROGER J. & GLEESON, JOHN \\
\hline AGEE, G. STEVEN & MACKINNON, GEORGE E. & WILKINS, WILLIAM W. \\
\hline WHITE, JEFFREY S. & FUSTE, JOSE ANTONIO & MURRAY, HERBERT F. \\
\hline DAVIS, EDWARD BERTRAND & JOHNSON, ALBERT WILLIAMS & VAN DUSEN, FRANCIS \\
\hline J P Stevens & R B Ginsburg & S A Alito \\
\hline PERRY, CATHERINE DELORES & GANEY, J. CULLEN & CAHILL, CLYDE S., JR. \\
\hline GIBSON, KIM R. & FORRESTER, J. OWEN & HARPER, ROY WINFIELD \\
\hline SNEED, JOSEPH T. & CHASE, HARRIE B. & ELLIOTT, JAMES ROBERT \\
\hline JENSEN, D. LOWELL & LEAVY, EDWARD & HIGGINS, THOMAS A. \\
\hline MCKEOWN, M. MARGARET & BEA, CARLOS T. & WEST, SAMUEL H. \\
\hline S D OConnor & S G Breyer & S Sotomayor \\
\hline BARRY, MARYANNE TRUMP & SUTTLE, DORWIN W. & ROBRENO, EDUARDO C. \\
\hline DECKER, BERNARD MARTIN & WOODS, GEORGE E., JR. & PICKERING, CHARLES WILLIS SR \\
\hline WILKINS, PHILIP C. & FAIRCHILD, THOMAS & NUGENT, DONALD C. \\
\hline BRIGGLE, CHARLES GUY & TEVRIZIAN, DICKRAN M., JR. & FARNAN, JOSEPH J., JR. \\
\hline DOOLING, MAURICE TIMOTHY & WEINFELD, EDWARD & LACEY, FREDERICK B. \\
\hline T Marshall & W H Rehnquist & W J Brennan \\
\hline VAN SICKLE, FREDERICK L. & MCAULIFFE, STEVEN JAMES & RESTANI, JANE A. \\
\hline COFFRIN, ALBERT $W$. & DUNCAN, ROBERT M. & YOUNG, GORDON E. \\
\hline BOOTLE, WILLIAM A. & KARLTON, LAWRENCE KATZ & NICHOLS, PHILIP, JR. \\
\hline MORTON, L. CLURE & GREEN, CLIFFORD SCOTT & MATSCH, RICHARD P. \\
\hline AGUILAR, ROBERT P. & MCNICHOLS, ROBERT J. & PUTNAM, WILLIAM LE BARON \\
\hline
\end{tabular}


Table 5: Most Similar Circuit Court Judges to Richard A. Posner

\begin{tabular}{|c|c|c|c|c|c|}
\hline Circuit Judge Name & Similarity & Rank & Circuit Judge Name & Similarity & Rank \\
\hline POSNER, RICHARD A. & 1.000 & 1 & TONE, PHILIP W. & 0.459 & 16 \\
\hline EASTERBROOK, FRANK $\mathrm{H}$. & 0.663 & 2 & SIBLEY, SAMUEL & 0.459 & 17 \\
\hline SUTTON, JEFFREY S. & 0.620 & 3 & SCALIA, ANTONIN & 0.456 & 18 \\
\hline NOONAN, JOHN T. & 0.596 & 4 & COLLOTON, STEVEN M. & 0.445 & 19 \\
\hline NELSON, DAVID A. & 0.592 & 5 & DUNIWAY, BENJAMIN & 0.438 & 20 \\
\hline CARNES, EDWARD E. & 0.567 & 6 & GIBBONS, JOHN J. & 0.422 & 21 \\
\hline FRIENDLY, HENRY & 0.566 & 7 & BOGGS, DANNY J. & 0.420 & 22 \\
\hline KOZINSKI, ALEX & 0.563 & 8 & BREYER, STEPHEN G. & 0.414 & 23 \\
\hline GORSUCH, NEIL M. & 0.559 & 9 & GOODRICH, HERBERT & 0.412 & 24 \\
\hline CHAMBERS, RICHARD H. & 0.546 & 10 & LOKEN, JAMES B. & 0.410 & 25 \\
\hline FERNANDEZ, FERDINAND F. & 0.503 & 11 & WEIS, JOSEPH F. & 0.408 & 26 \\
\hline EDMONDSON, JAMES L. & 0.501 & 12 & SCALIA, ANTONIN (SCOTUS) & 0.406 & 27 \\
\hline KLEINFELD, ANDREW J. & 0.491 & 13 & BOUDIN, MICHAEL & 0.403 & 28 \\
\hline WILLIAMS, STEPHEN F. & 0.481 & 14 & RANDOLPH, A. RAYMOND & 0.397 & 29 \\
\hline KETHLEDGE, RAYMOND M. & 0.459 & 15 & MCCONNELL, MICHAEL W. & 0.390 & 30 \\
\hline
\end{tabular}

bundle of citations to precedents, in the same way that Rudolph et al. (2017) treat grocery baskets as a bundle of products. The model predicts the presence of a particular citation using the list of co-occurring citations. As with word embeddings, cases that tend to be cited together locate near each other in the embedding space. In consequence, the model serves to locate cases in a "precedent space" as opposed to a language space. An intriguing feature is that the learned parameters encode complentarity or substitutability of items; in the context of Rudolph et al. (2017), that means coffee being substitutable with tea but complementary with milk, for example. In the context of the law, we learn what precedents tend to be cited together, and which tend to be substitutes. By pairing substitutability metrics with ideological valence (liberal versus conservative), we can analyze the parallel histories of liberal and conservative jurisprudence in the United States.

\subsection{Language-Based Metrics of Implicit Bias}

Another future avenue in this area is the use of embeddings to extract sentiment or bias in judicial language. The work of Caliskan et al. (2017), who use an off-the-shelf word embeddings model GloVe, is a natural starting place. This pre-trained word embedding 
provides a representation of English-language words in a 300-dimensional vector space. They then compute similarity, which means having the same direction in the word vector space, between groups of words. In Ash et al. (2018a), we apply this approach to judicial decision language.

To summarize, we start with a set of sentiment words. These could include, for example, a set of words with positive sentiment ("good", "best", "pleasant", ...) and a set of words with negative sentiment ("bad", "worst", "unpleasant", ...). We take the average vector for the positive words, "pleasant" $\left(\vec{w}_{+}\right)$, and the average vector for the negative words, "unpleasant" $\left(\vec{w}_{-}\right)$. The idea is that the average of these vectors encodes the shared semantic component between these words for positive and negative. This shared component is likely a more accurate representation or location of these concepts in the language space. In addition, because the word embeddings are trained on the legal corpus, they are valid to this context.

Next, we have a set of words identifying some social distinction, such as race. The vector for "white" $\left(\vec{w}_{W}\right)$ might include "european", "caucasian", etc., while the vector for "black" $\left(\vec{w}_{B}\right)$ might include "african", "afro-american", etc. We then have an average vector for each social group, with the idea that the "concept" of these social groups is more accurately located in the language space. Another way to do this is to get the average vectors for names that are disproportionately given to white and black individuals (Caliskan et al., 2017). This may not work in a legal context where first names are not used very often.

Next, one can compute the cosine similarity between the two sentiment categories on the one hand, and the two social-group categories on the other. Using these metrics, on could construct a "word embedding association test" (analogous to the "implicit association test" from psychology studies) using

$$
\begin{aligned}
\text { Word Embedding Association Test } & =\frac{\text { White-Pleasant Association }}{\text { White-Unpleasant Association }}-\frac{\text { Black-Pleasant Association }}{\text { Black-Unleasant Association }} \\
& =\frac{s\left(\vec{w}_{W}, \vec{w}_{+}\right)}{s\left(\vec{w}_{W}, \vec{w}_{-}\right)}-\frac{s\left(\vec{w}_{B}, \vec{w}_{+}\right)}{s\left(\vec{w}_{B}, \vec{w}_{-}\right)}
\end{aligned}
$$

where $s(\cdot)$ is cosine similarity. A positive value to this test means that positive-sentiment language is more closely associated to the white race, relative to the black race, in the corpus on which the word embeddings are trained. Caliskan et al. (2017) show that in a set of word embeddings trained on a broad corpus of English, there is a significant relative white-positive relation.

These types of metrics could be potentially applied to legal writings. The idea is that the text of a judge's opinions could be used to detect variation in implicit bias across judges. 
We could ask, for example, whether judges with a lexical bias against blacks also tend to reject discrimination complaints, or to give longer criminal sentences to blacks. We could also look for peer effects, and see whether sitting with a biased judge has an impact on a judge's decisions.

There are broader applications of lexical association available. For example, we could look at relative positive sentiment toward particular types of policies, and see whether that is associated with policy choices of the judges. We could look at gender stereotype associations, for example associating doctor with male and nurse with female. Having more traditional gender views, as detected in one's implicit language bias, might be reflected in more conservative judicial decisions related to gender rights, such as equal employment cases and gender discrimination cases.

\subsection{Judge Embeddings}

We saw in Section 3 that document embeddings trained from a word prediction task did not do a good job of discriminating judges on ideology. A major factor in this limitation is that the embeddings are trained just from language style of written decisions. They do not account for the direction of the decision (e.g., for or against plaintiffs). Perhaps more importantly, they do not account for the lower-court decision features. In this subsection we outline a targeted approach that could address these shortcomings.

To be more precise, we can move forward with the deep learning literature and directly implement an embedding layer for judge identity. Word embeddings are constructed by locating words together that are most similarly predictive for a deep learning task. In the same way, a judge embedding could be learned by a deep learning model which locates judges together that are similarly impactful in a machine prediction task. One can use richer representations of judge characteristics besides their language, including the directions of their decisions and their citations to previous opinions. Moreover, one can let the impact of these features interact with the features of the lower-court decision being considered.

Consider the following model of judical opinion generation. The unit of observation is an opinion $i$, written by judge $j$ at time $t$ in court/jurisdiction $c$. The opinion is a matrix of features $Y_{i}$, including the ruling (affirm/reverse), the text features of the opinion, and the set of citations to previous opinions. The case is a review of a district court opinion, represented by a vector of features $D_{i}$, including the text and metadata from the district court. A set of controls $X_{c t}$ includes a range of characteristics for court and time, including some measure of the stock of precedents in court $c$ at time $t$. 
We would like to predict $Y_{i}$ by approximating

$$
Y_{i} \sim F\left(D_{i}, X_{c t}, j\right)
$$

where $F(\cdot)$ is some distribution over opinion features we can approximate using a deep neural net (e.g. Goodfellow et al., 2016). Unlike the regression models that most empirical legal scholars are used to, neural nets can easily accommodate high-dimensional outcomes (such as $Y_{i}$ ). The model would be trained by backpropagation with stochastic gradient descent.

In particular - and this is the key innovation - the judge identity $j$ will be represented with an embedding lookup layer to a relatively low-dimensional dense vector space. The location of the judge vectors, initialized randomly, would be endogenous to the model. As the model goes through further training, the locations of these vectors will be pushed around to improve predictiveness. As a by-product of the model, the judges that locate together in the vector space would be predicted to behave similarly on the court holding other factors equal.

This model could then be used to simulate counterfactuals. For example, how would the decision in a case change by switching out the authoring judge $j$ ? How would the style of language change for a different circuit $c$ ? This will give us new insight into the topography of ideology in the U.S. judiciary.

\section{References}

Ash, E. (2016). The political economy of tax laws in the u.s. states. Technical report. 2

Ash, E. (2017). Emerging tools for a 'driverless' legal system: Comment. Journal of Institutional and Theoretical Economics. 2

Ash, E., Chen, D., and Liu, W. (2017). The (non-)polarization of u.s. circuit court judges, 1930-2013. Technical report. 3.4, 4.2

Ash, E., Chen, D. L., and Ornaghi, A. (2018a). Implicit bias in the judiciary: Evidence from judicial language associations. Technical report. 4.1, 4.3

Ash, E., MacLeod, W. B., and Naidu, S. (2018b). Optimal contract design in the wild: Rigidity and discretion in collective bargaining. Technical report. 1 
Blei, D. M. (2012). Probabilistic topic models. Communications of the ACM, 55(4):77-84. 1,2

Bolukbasi, T., Chang, K.-W., Zou, J. Y., Saligrama, V., and Kalai, A. T. (2016). Man is to computer programmer as woman is to homemaker? debiasing word embeddings. In Advances in Neural Information Processing Systems, pages 4349-4357. 2

Breyer, S. (2009). Economic reasoning and judicial review. Economic Journal, 119(535):F215-F135. 3.5

Caliskan, A., Bryson, J. J., and Narayanan, A. (2017). Semantics derived automatically from language corpora contain human-like biases. Science, 356(6334):183-186. 4.3

Cameron, C. and Kornhauser, L. (2017). What courts do ... and how to model it. Technical report, NYU Law and Economics Research Paper. 1

GangImair, B. and Wardlaw, M. (2017). Complexity, standardization, and the design of loan agreements. Technical report. 1

Goodfellow, I., Bengio, Y., and Courville, A. (2016). Deep Learning. MIT Press. http: //www. deeplearningbook. org. 4.4

Grover, A. and Leskovec, J. (2016). Node2vec: Scalable feature learning for networks. In Proceedings of the 22Nd ACM SIGKDD International Conference on Knowledge Discovery and Data Mining, KDD '16, pages 855-864, New York, NY, USA. ACM. 4.2

Jurafsky, D. and Martin, J. H. (2014). Speech and language processing, volume 3. Pearson London. 1

Le, Q. and Mikolov, T. (2014). Distributed representations of sentences and documents. In Proceedings of the 31st International Conference on Machine Learning, volume 32 of Proceedings of Machine Learning Research. 1, 2, 3.2

Leibon, G., Livermore, M., Harder, R., Riddell, A., and Rockmore, D. (2018). Bending the law: geometric tools for quantifying influence in the multinetwork of legal opinions. Artificial Intelligence and Law, 26(2):145-167. 1

Levy, O. and Goldberg, Y. (2014). Dependencybased word embeddings. In Proceedings of the 52nd Annual Meeting of the Association for Computational Linguistics, volume 2, pages 302-308. 3.4 
Levy, O., Goldberg, Y., and Dagan, I. (2015). Improving distributional similarity with lessons learned from word embeddings. Transactions of the Association for Computational Linguistics, 3:211-225. 1, 2

Livermore, M. A., Riddell, A., and Rockmore, D. (2016). Agenda formation and the us supreme court: A topic model approach. Arizona Law Review. 1

Maaten, L. v. d. and Hinton, G. (2008). Visualizing data using t-sne. Journal of Machine Learning Research, 9(Nov):2579-2605. 3.4

Mikolov, T., Sutskever, I., Chen, K., Corrado, G. S., and Dean, J. (2013). Distributed representations of words and phrases and their compositionality. In Advances in neural information processing systems, pages 3111-3119. 1, 2

Rudolph, M. and Blei, D. (2017). Dynamic bernoulli embeddings for language evolution. arXiv preprint arXiv:1703.08052. 4.1

Rudolph, M., Ruiz, F., Athey, S., and Blei, D. (2017). Structured embedding models for grouped data. In Advances in Neural Information Processing Systems, pages 250-260. 2, $4.1,4.2$

Songer, D. R. and Haire, S. (1992). Integrating alternative approaches to the study of judicial voting: Obscenity cases in the u.s. courts of appeals. American Journal of Political Science, 36(4):963-982. 1 